\title{
Wrist Actimetry Biomarker Development of Paretic Upper Limb Use in Post Stroke Patients for Ecological Monitoring.
}

\section{Gilles Dusfour ( $\nabla$ g-dusfour@chu-montpellier.fr)}

University of Montpellier: Universite de Montpellier https://orcid.org/0000-0002-1388-3135

\section{Denis Mottet}

Université de Montpellier: Universite de Montpellier

\section{Makii Muthalib}

University of Montpellier: Universite de Montpellier

Isabelle Laffont

University of Montpellier: Universite de Montpellier

Karima K.A. Bakhti

University of Montpellier: Universite de Montpellier

\section{Research}

Keywords: Actimetry, Stroke, hemiparesis, biomarkers, upper limb

Posted Date: September 17th, 2021

DOI: https://doi.org/10.21203/rs.3.rs-845425/v1

License: (c) (i) This work is licensed under a Creative Commons Attribution 4.0 International License. Read Full License 
1 Wrist actimetry biomarker development of paretic upper limb use in post stroke patients for ecological monitoring.

4 Gilles Dusfour ${ }^{1}$, Denis Mottet $^{2}$, Makii Muthalib ${ }^{2,3}$, Isabelle Laffont ${ }^{1,2,3}$, Karima K.A. Bakhti ${ }^{2,3}$

61 : CARTIGEN, CHU de Montpellier, 34090 Montpellier, France

7 2: University of Montpellier, Euromov Digital Health in Motion, IMT Mines Alès, 8 Montpellier, France

9 3: Physical and Rehabilitation Medicine, Montpellier University Hospital, University of 10 Montpellier, Euromov Digital Health in Motion, Montpellier, France

12 Corresponding author: Gilles Dusfour (g-dusfour@chu-montpellier.fr)

\section{Abstract}

15 Background

16 In post-stroke patients it is unclear which wrist actimetry biomarkers to use to estimate the

17 degree of upper limb hemiparesis. The objective of this study was to develop a general and

18 objective framework for monitoring hemiparetic patients in their home environment via

19 different biomarkers based on 7 days of actimetry data. A secondary objective was to use all of 
these biomarkers to better understand the mechanism for potential non-use of the paretic upper

21 limb.

\section{Methods}

Accelerometers were worn continuously for a period of 7 days on both wrists of 10 post-stroke hemiparetic patients as well as 6 healthy subjects. Various wrist actimetry biomarkers were calculated, including the Jerk ratio 50 (JR50, cumulative probability that the Jerk Ratio is between 0 and 0.5 ), absolute and relative amounts of functional use of movements of the upper limbs (FuncUse and FuncUseR) and absolute and relative velocities of the upper limbs during functional use (VUL and VULR). For each biomarker, the values of stroke and healthy groups were compared. The correlations between all the biomarkers were studied.

\section{Results}

We studied 10 participants with mild-to-moderate chronic hemiparesis and 6 healthy control participants. FuncUse and VUL of the paretic upper limb of stroke patients were significantly lower than in the non-dominant upper limb of healthy subjects. Similarly, FuncUseR (paretic/non-paretic vs non-dominant/dominant), JR and VULR are significantly lower in stroke patients than in healthy subjects. FuncUseR, VULR and JR50 seem to be complementary biomarkers for monitoring patient strokes.

\section{Conclusion}

The stroke patients do not seem to compensate for the decrease in functional movement on the paretic side by an increase on the non-paretic side. The speed of execution of functional movements on the paretic side could be the limiting factor to a normal use of the paretic upper limb. A thorough clinical study is needed to identify the limiting factors. In conclusion, this study for the first time has shown actimetry is a robust and non-obtrusive lightweight 
technology for continuously acquiring objective upper limb data of paretic arm use/ non-use over an extended period in a home environment for monitoring stroke patients.

Keywords: Actimetry, Stroke, hemiparesis, biomarkers, upper limb,

\section{Background}

Stroke is one of the leading causes of disability worldwide, with a global prevalence rate that has been increasing over the past 30 years [Murray et al., 2012]. Despite the accumulated research on rehabilitation of the upper limb (UL) following a stroke, a large majority of patients continue to present non-use of paretic upper limb at the chronic stage which impacts their quality of daily life [Morris et al, 2013]. As such, only 5 to $20 \%$ of stroke survivors regain UL function after 6 months [Kwakkel et al, 2003]. Although there are numerous clinically based assessments of paretic arm use/non-use, objective, robust, and reproducible indicators of the amount of UL use in a home environment are needed for better monitoring the paretic UL use and non-use and the response to various proposed treatments aiming at improving motricity and functioning.

Current methods of quantifying movement of the upper limbs rely primarily on clinical deficit scores such as the Fugl-Meyer test [Fugl-Meyer et al, 1975], or on more functional tests like Wolf Motor Function Test (WMFT), Action Research Arm Test (ARAT) or questionnaires (Motor Activity Log - MAL). A more recent work focused on the direct visual observation of stroke patients by hospital practitioners in a clinical environment during 7 days [McLaren et al, 2020]. This work found that the ratio of use activity between the paretic limb and the nonparetic limb is around 0.69 for stroke patients [McLaren et al, 2020] whereas it is 0.95 for 
healthy subjects (non-dominant/dominant) [Bailey et al, 2014]. The human assessor method used by McLaren, [McLaren et al, 2020] has the advantage of identifying with certainty the periods of functional use as assessed directly by the clinician. However, the time and human resources costs of performing these measurements reduce its applicability to monitor multiple patients, and moreover, limiting observations in a clinical setting and not in a home environment reduces the ecological validity of these observations.

Alternatively, a commonly used quantitative and objective technique to quantify functional UL movements relies on methods based on actimeters or gyroscopes [Bailey et al, 2014] positioned on the wrists over a period of time ranging from 2 to 7 days. The functional UL movement results of Bailey's work [Bailey et al, 2014; Bailey et al, 2015] are based on the calculation of activity counts directly from the acceleration signals. The authors then obtain activity durations and intensities. However, these metrics have shown limitations, especially since the proprietary activity count algorithms do not allow for validation and standardization of the method. To overcome this, Pan et al, [Pan et al, 2020] developed new accelerometric biomarker based on the Jerk, which is the derivative of acceleration. He showed that the Jerk ratio (JR) has a very high sensitivity to the amount of UL motion as well as a very high correlation with the biomarkers developed by Bailey et al. Leuenberger [Leuenberger et al, 2017] extended the method by using inertial sensors (accelerometer and gyroscope) as inclinometers. This allowed the authors to define functional upper limb movements according to elevation angle and range of motion in a given time space. Leuenberger [Leuenberger et al, 2017] found excellent correlation of these biomarkers with the box and block test. However, Leuenberger's work [Leuenberger et al, 2017] is based on inertial sensors with low energy autonomy, which only allow for measurements over 2 consecutive days. In addition, no comparison was made with healthy subjects. 
In this study, we developed a new method to derive a biomarker of functional UL use using two accelerometers positioned at each wrist that couples the calculation of the JR with the elevation angle of the UL over a period of 7 days, in the patients' ecological environment. The new biomarker is termed the execution velocity of functional upper limbs (VUL) movements that is calculated via the temporal derivative of the elevation angle of the UL. We then compared the different accelerometric biomarkers between a population of 10 stroke patients and 6 healthy subjects.

\section{Methods}

\section{Participants}

In this study, a sample of 10 stroke survivors and a sample of 6 healthy subjects were recruited by the Physical and Rehabilitation Medicine PRM) department of Montpellier University Hospital. Each participant was asked to sign an informed consent form approved by the Institutional Review Board (the local ethics commission). Patients were recruited in the PRM unit between December 2019 and May 2021. The post-stroke participants met the following inclusion criteria: (1) diagnostic criteria for stroke, (2) people after an ischemic or haemorrhagic stroke that suffered from a paretic arm (defined as a Fugl Meyer -Upper Extremity - FM-UE score $>15 / 66$ ), in the chronic stage of recovery (>6month post-stroke). (2) 18 years or older. The exclusion criteria were the following: (1) Mini-Mental Status Examination score <24 [Bleecker et al, 1988], (2) strong neglect with a Bell's test $>15$ bells (3) othopedic or rheumatologic injury on the forearm, (3) pregnancy. The controls had no self-reported injuries that would alter or impair their use of either UL.

\section{Procedures}

Accelerometers (Axivity Ax3, Newcastle upon Tyme, UK) were placed on each wrist for all participants. The patients were asked to wear the accelerometers for 7 days without removing them. Data acquisition was performed at a frequency of $50 \mathrm{~Hz}$ coupled with a cut off of $8 \mathrm{~g}$ for 
114 the measurement of acceleration in the three spatial directions. The accelerometers were

115 recovered at the end of the 7 days to extract the data using the OmGui software provided by

116 Axivity. The data were sliced day by day to obtain daily acceleration data values. The data were

117 then saved in csv format so they can be read by any programming language.

\section{Biomarkers}

119 Data processing was done using the python 3.7 programming language. The numpy and scipy

120 libraries are notably used for numerical calculation operations (derivation, frequency analysis).

121 The scipy library allows the application of a low pass filter with a cut-off frequency of $10 \mathrm{~Hz}$ in

122 order to remove noise. The magnitude of the acceleration vector (SVM: scalar vector

123 magnitude) is then calculated for each time step of the two actimeters (via the acceleration data

124 at a given time $\left.t: a_{x}(t) ; a_{y}(t) ; a_{z}(t)\right)$.

$$
\operatorname{svm}(\mathrm{t})=\sqrt{\mathrm{a}_{\mathrm{x}}^{2}+\mathrm{a}_{\mathrm{y}}^{2}+\mathrm{a}_{\mathrm{z}}^{2}}
$$

128 The time derivative of the acceleration at a given time $t$ allows us to obtain the Jerk, noted $\mathrm{J}$, in

129 the three directions of space via the following calculation (finite difference centered 130 approximation):

$$
J_{i}(t)=\frac{a_{i}(t+d t)-a_{i}(t-d t)}{2 d t}
$$

133 Where $i$ represents the three directions of space $x, y$ and $z, a$ is the scalar value of the acceleration

134 and dt the sampling time step (i.e. 50Hz). Physically, the Jerk represents the rate of change of

135 the acceleration vector. It is then possible to calculate the magnitude of the Jerk: 


$$
\operatorname{Jerk}_{M a g}=\sqrt{J_{x}^{2}(t)+J_{y}^{2}(t)+J_{z}^{2}(t)}
$$

137 Pan et al., [Pan,2020] showed that the jerk ratio (JR) is sensitive to the degree of upper limb

138 mobility. The jerk ratio is defined as the ratio of the jerk amplitude of the paretic (non-dominant)

139 limb to the sum of the jerk amplitude of the paretic (non-dominant) limb and the nonparetic

140 (dominant)

141 limb:

$$
\operatorname{Jerk}_{\text {Ratio }}=\frac{\mid \text { Jerk }_{\text {non-paretic }} \mid}{\mid \text { Jerk }_{\text {paretic }}|+| \text { Jerk }_{\text {non-paretic }} \mid}
$$

143 Points where the jerk of the paretic or non-paretic side is equal to zero are excluded from the

144 study. A JR close to 0 means a preponderant use of the paretic (non-dominant) arm while a jerk

145 ratio close to 1 means a preponderant use of the non-paretic (dominant) arm. It is then possible

146 to calculate the histogram and probability density function of the JR for each measurement day.

147 The probability density function is normalised to give a total probability distribution of 1.

148 Following the work of Pan et al, [Pan et al, 2020], the jerk ratio 50 (JR50) was calculated. This

149 metric corresponds to the cumulative probability that the JR is between 0 and 0.5 . A JR50 value

150 greater than 0.5 suggests a preponderant non-paretic (dominant) arm mobility.

152 In quasi-static condition, the calculation of the angle of elevation of the forearm with respect to

153 the gravity vector takes the form of equation 6, following the trigonometric laws:

$$
\alpha(t)=\operatorname{arcos}\left(\frac{a_{y}(t)}{\operatorname{svm}(t)}\right)
$$


155 It is then possible to obtain the angular velocity of elevation by the time 156 derivative:

$$
\dot{\alpha}(\mathrm{t})=\frac{\alpha(t+d t)-\alpha(t-d t)}{2 d t}
$$

161 Leuenberger et al., 2017, [Leuenberger et al., 2017] estimates that the upper limbs perform a

162 functional movement when there is a variation in the angle of inclination of the arm greater 163 than $30^{\circ}$ and that this same angle of inclination is between $\pm 30^{\circ}$ (to avoid data from walking)

164 all within a time window of 0.5 seconds. The mathematical formulation is as follows:

$$
|\alpha| \leq 30^{\circ} \text { and } \alpha_{\max }-\alpha_{\min } \geq 30^{\circ}
$$

166 A functional movement iteration counter is created for both upper limbs for each day. The

167 counter is updated for each functional movement detected. The absolute values of functional movements and ratio (paretic/non-paretic or non-dominant/dominant) are presented as a boxplot with the median value of the 7 days of measurements.

173 Each biomarker was qualitatively compared between the post-stroke population and the healthy

174 population using boxplots. Depending on the normality or not of the data distribution, identify

175 by the Shapiro test, student test or non-parametric Wilcoxon-Mann-Whitney test was applied.

176 Scatter plots were performed to visualise the relationships between the ratio of upper limb use 8 
177 or the number of movements on the paretic arm with all the calculated biomarkers. Depending

178 on the distribution of the scatter plot data, linear relationships were established between the

179 upper limb ratio or the number of movements on the paretic side with the rest of the biomarkers.

180 The coefficient of determination is computed to assess the goodness of the fit with the

181 experimental data. Regarding the large number of biomarkers, principal component analysis

182 (PCA) was used for its potential for data reduction and explanation. To overcome the different

183 units of measurement, the data were standardized. Then only the first two principal components

184 were selected to explain the results.

Table 1: Stroke patients and healthy subject characteristics

\begin{tabular}{|l|c|c|}
\hline \multicolumn{2}{|c|}{ Post Stroke patients } & Healthy volunteers \\
\hline Number & 10 & 6 \\
\hline Age in years & $67 \pm 12[47-82]$ & $45 \pm 18[18-75]$ \\
\hline Gender & 4 males, 6 females & 3 males -3 females \\
\hline Affected body side & 5 right, 5 left & - \\
\hline Dominant Side Affected & $5(50 \%)$ & - \\
\hline FM-UE Score (/66) & $50.5 \pm 14[27-66]$ & - \\
\hline
\end{tabular}

190

\section{Jerk Ratio}

192 Figure 1.A shows the histogram and probability density function (PDF) of the JR for a healthy

193 subject on a representative day. We can see that the histogram is centered on a value of 0.5 ,

194 which highlights a balance in the movement of the upper limbs. A slight peak can also be seen

195 at a JR value of 0 and 1, highlighting a non-negligible amount of probability of movement of

196 the dominant limb only or non-dominant limb only, respectively. Figure 1.B compares the one9 
198 stroke patient is positioned at a value of 0.9, highlighting a preponderance of movement of the non-paretic limb. Figure 1.C compares the group median JR50 values between the two populations using a boxplot. The post-stroke population has a median JR50 value of 0.55 which is significantly higher than the median value of 0.51 for healthy subjects ( $t$-test $\mathrm{p}<0.05)$. In addition, there is a very high inter-patient variability in the stroke population, indeed the range and 0.53 .
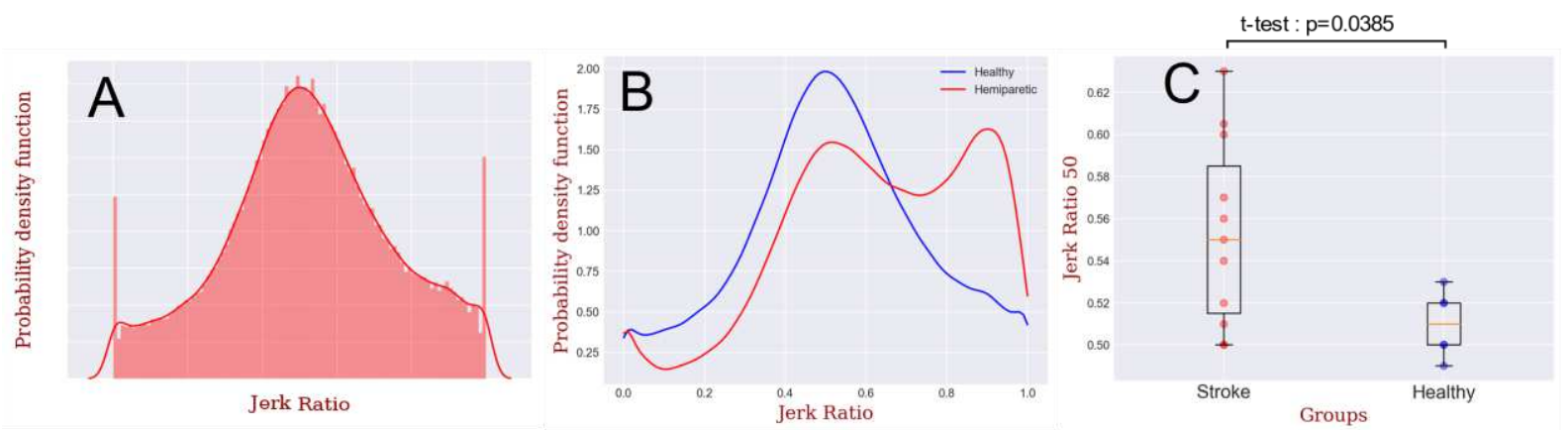

Figure 1: (A) Normalized probability density function of the Jerk ratio JR of a healthy subject. A JR of 0 indicates use of the

paretic (non-dominant) limb and a ratio of 1 indicates use of the non-paretic (dominant) limb. (B) Comparison of the $J R$

probability density functions of a healthy subject and a stroke patient. The healthy subject has a maximum probability for a

$J R$ of 0.5 (use of both limbs at the same time) while the maximum probability of the JR for the stroke patient is 0.9 (predominant

use of the non-paretic limb). (C) Boxplot of the median JerkRatio50 (JR50) values for the stroke and healthy groups. Each point corresponds to the median JR50 value of each subject ( $t$ test: $p$ value $=0.0385$ ).

213 Figure 2.A shows the median number of functional movements of the paretic (non-dominant) 214 and the non-paretic (dominant) upper limb over a 7-day period for the stroke and healthy participants. It can be observed that the median values of functional movements (FuncUse) of the paretic upper limb of stroke patients (median: 1500, range: [0; 3500] movements) were

217 significantly lower (WMW test: $\mathrm{p}<0.001)$ than the values of the non-dominant limb of healthy 10 
218 subjects' movements (median: 5000, range: [4500; 9000]). In contrast, stroke patients 219 compensate with the non-paretic limb where they can reach a median 6000 movements in one 220 day (range: $[1500,13000]$ ) (fig 2.B). Figure 2.C shows the boxplots of the median FuncUseR 221 of the paretic upper limb to the non-paretic upper limb for the stroke and healthy groups (ratio 222 of dominant/non-dominant UL). It can be seen that the median ratio over 7 days of measurement 223 was significantly lower (WMW test $\mathrm{p}<0.005)$ for the stroke (0 to $0.5,0$ to 50 movements of the 224 paretic limb per 100 of the nonparetic limb) than for the healthy (0.6 to 1.3 , 60 to 130 movements of the non-dominant limb per 100 of the dominant limb) population.

226

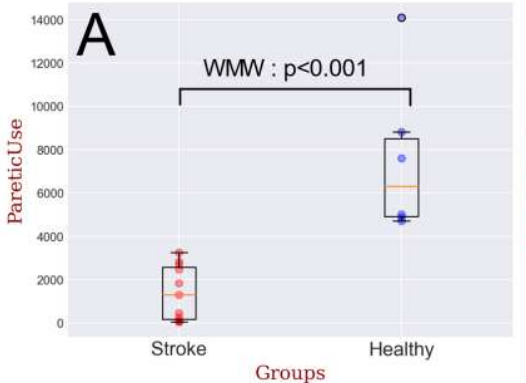

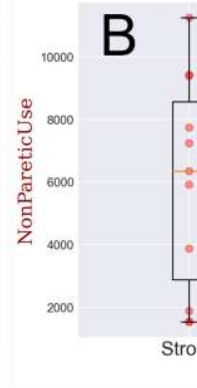

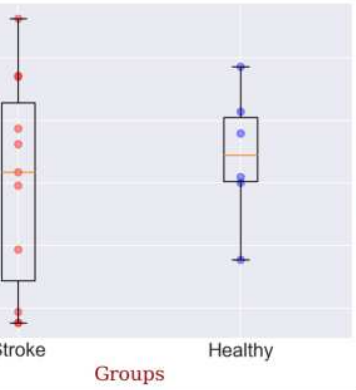

Figure 2: Boxplot of the median functional use of movements (FuncUse) of the (A) paretic and non-dominant (B) non-paretic and dominant UL of the stroke and healthy groups, respectively. (C) Boxplot of the median functional use of movement ratio (FuncUseR) between UL of the stroke (paretic/non-paretic) and health (non-dominant/dominant) groups. As shown in Fig.1A, healthy subjects show a larger number of FuncUse of the non-dominant UL than stroke patients paretic UL. As shown in Fig2B, healthy subjects and stroke patients show a comparable amount of FuncUse of the non-paretic and dominant UL, respectively. As shown in Fig2C, the FuncUseR of the healthy subjects were larger than stroke patients.

\section{Functional movement elevation speed}

Fig.3a shows the functional movement elevation velocities of the UL (VUL) for the stroke and healthy groups. In figure 3.A we can observe that the VUL of healthy subjects on the nondominant side are significantly higher $(\mathrm{p}<0.05)$ than the stroke patients on the paretic side. Indeed, the median VUL over 7 days of measurements are between 135 and 190 for the healthy 
subjects and between 110 and 160 for the stroke patients (see figure 3.A, WMW test: $\mathrm{p}<0.05$ ).

240 The VUL of the stroke patients on their non-paretic side were significantly lower than the

241 healthy subjects on their dominant side (Figure 3.B: $\mathrm{p}<0.05$ ). For the stroke patients, the VUL

242 ranged from 105 to 159 and from 138 to 175 for healthy subjects (see figure 3.B). Figure 3.C

243 presents the VULR of paretic/non-paretic (non-dominant/dominant) for the stroke and healthy

244 population. It can be observed for the stroke patients that the VULR of the paretic limb were $24510 \%$ lower than those of the non-paretic limbs (i.e. speed ratio of 0.83 to 1.22 ). In comparison, 246 most of the VULR of healthy subjects were greater than 1 (speed ratio interval of $[0.95 ; 1.12]$ ) 247 (WMW test: $\mathrm{p}<0.05)$.
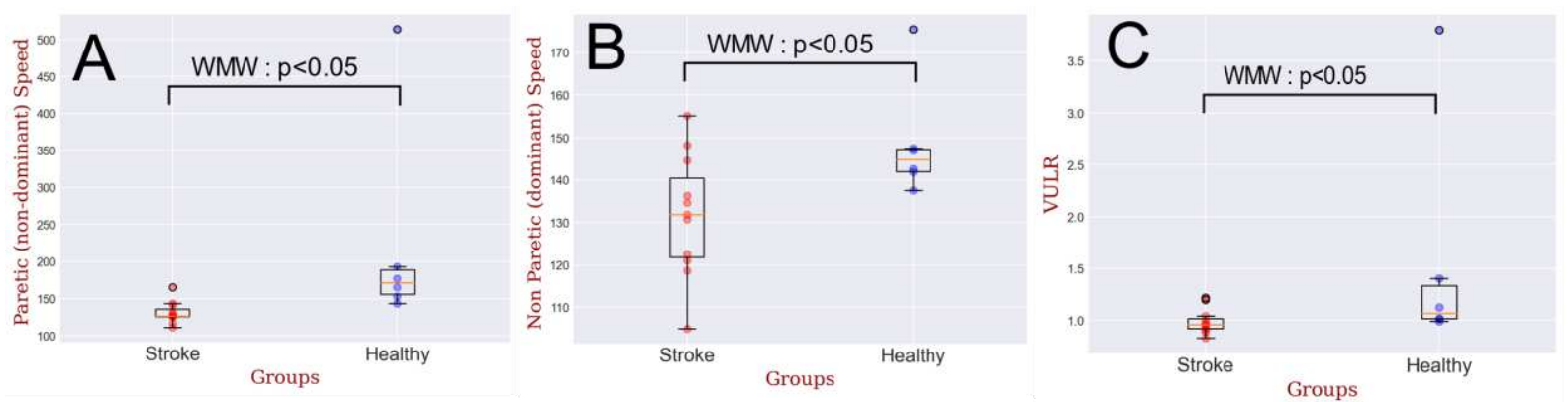

Figure 3: Boxplot of median functional movement elevation speed of the UL (VUL) for the stroke and healthy groups: (A) Paretic

vs non-dominant UL, (B) Non-Paretic vs dominant UL, (C) Ratio of UL velocity (VULR). In Fig3a healthy subjects show a greater

255 Figure 4.A shows the linear relationship between FuncUse on the paretic side and the FuncUseR 256 for stroke patients $\left(\mathrm{r}^{2}=0.36, \mathrm{p}<0.001\right)$. Indeed, the more a stroke patient tends to use his/her paretic upper limb, the more the FuncUseR tends towards 1. This relationship does not exist for healthy subjects (Fig4a; $\mathrm{p}>0.05$ ). In parallel, Figure 4.B shows that there is a linear relationship 
between the amount of FuncUse of the dominant and non-dominant upper limbs for healthy 260 subjects $\left(\mathrm{r}^{2}=0.49, \mathrm{p}<0.001\right)$ whereas this is not the case for stroke patients considering the 261 paretic and non-paretic UL. This means that for healthy subjects, the more they use their 262 dominant limbs the more they use their non-dominant limbs. Furthermore, a very strong 263 correlation was found between VULR and FuncUseR for healthy subjects (Figure 4.C, $\mathrm{r}^{2}=0.8$, $264 \mathrm{p}<0.001)$ but not for stroke patients. This relationship shows that the VULR must reach a value 265 of 1.1 for a healthy subject to have a FuncUseR of 1 . At the same time, stroke patients have 266 very high VULR (1.75) without the FuncUseR exceeding 0.25. Finally, Figure 4.D highlights 267 the relationship between two biomarkers of the amount of upper limb functional movement use, 268 the FuncUseR and the JR50. Figure 4.D shows a curve of decreasing exponential appearance 269 where as JR50 increases the FuncUseR decreases. This graph shows the greater sensitivity of 270 the FuncUseR for healthy subjects. Indeed, while the JR50 varies between 0.48 and 0.54 for 271 healthy subjects, the FuncUseR varies in a range from 0.5 to 1.5 . On the other hand, the JR50 272 has a very high sensitivity for subjects with very little movement on the paretic side. Notably, 273 a stroke patient presents a FuncUseR between 0 and 0.05 while his JR50 varies in a range of $274 \quad 0.54$ to 0.77 

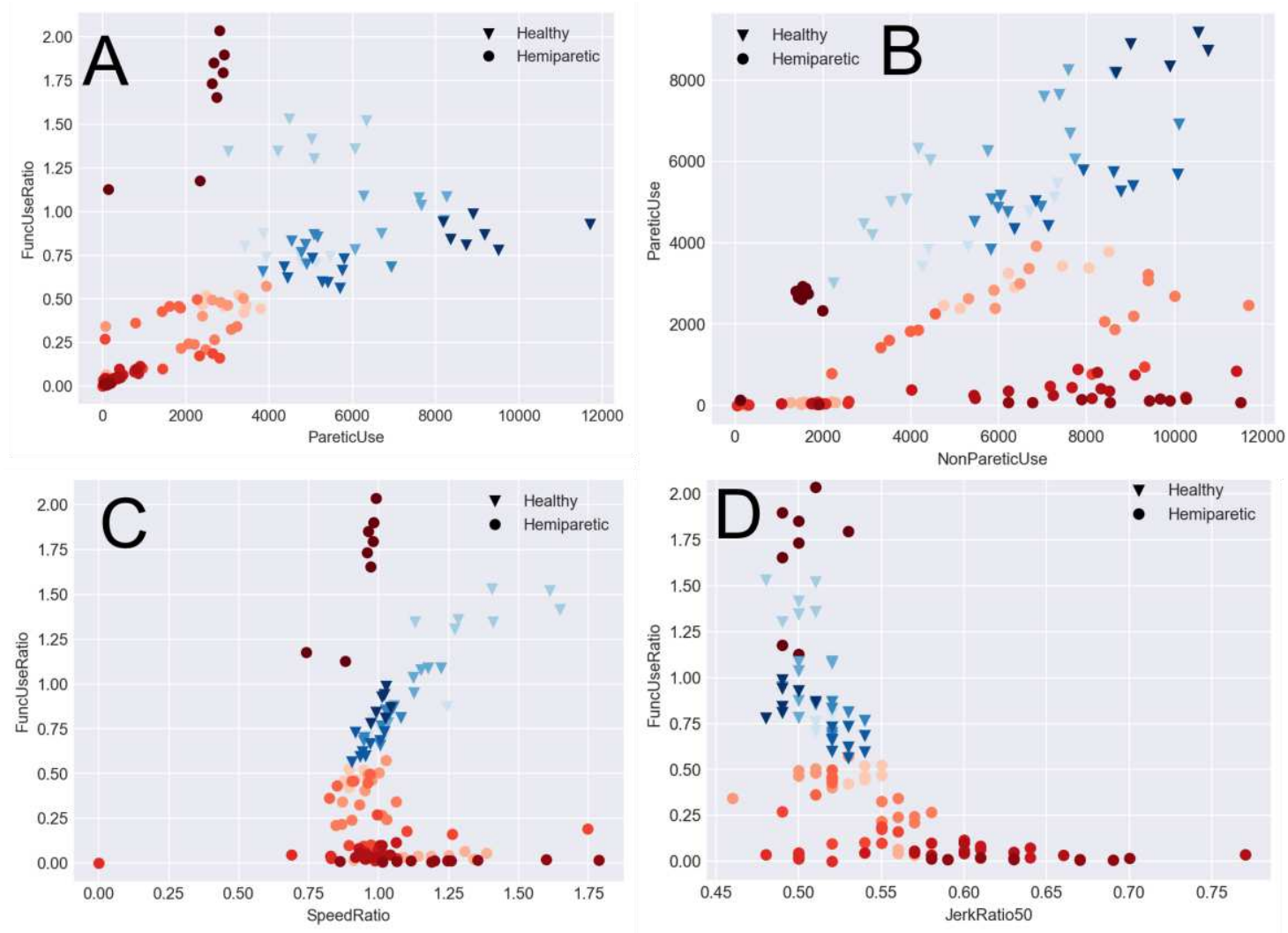

Figure 4: Relationship between functional use of movement of the UL (FuncUse) to derived biomarkers. (A) FuncUseR

relationship to FuncUse on the paretic side for stroke patients and non-dominant side for the healthy subjects. (B) FuncUse of

the paretic (non-dominant) side in relation to the non-paretic (dominant) side for the stroke (healthy) group. (C) (FuncUseR

relationship to VULR. (D) FunCUseR relationship to JR50. Healthy subjects are represented by blue circles and stroke patients

by red triangles with a colour gradient differentiating subjects.

\section{Principal component analysis}

The different biomarker principal component analysis showed that principal components 1 and 2 (PC1 and PC2) accounted for $51 \%$ and $24 \%$ of the variance in the results, i.e. $75 \%$ in total. Figure 5. A shows the position of each study participant in relation to PC1 and PC2. The healthy subjects all have positive $P C 1$ values while the stroke patients all have negative PC1 values except for one subject with a FuglMeyer of 66. Figures 5.B and 5.C show the relative importance of each biomarker in PC1 and PC2 respectively. We see the two most important biomarkers in CP1 are related to the FuncUse and VUL of use of the paretic limb while the most important biomarkers in CP2 are related to the FuncUse of the non-paretic limb and the JR50. 


\section{Principal Component Analysis}

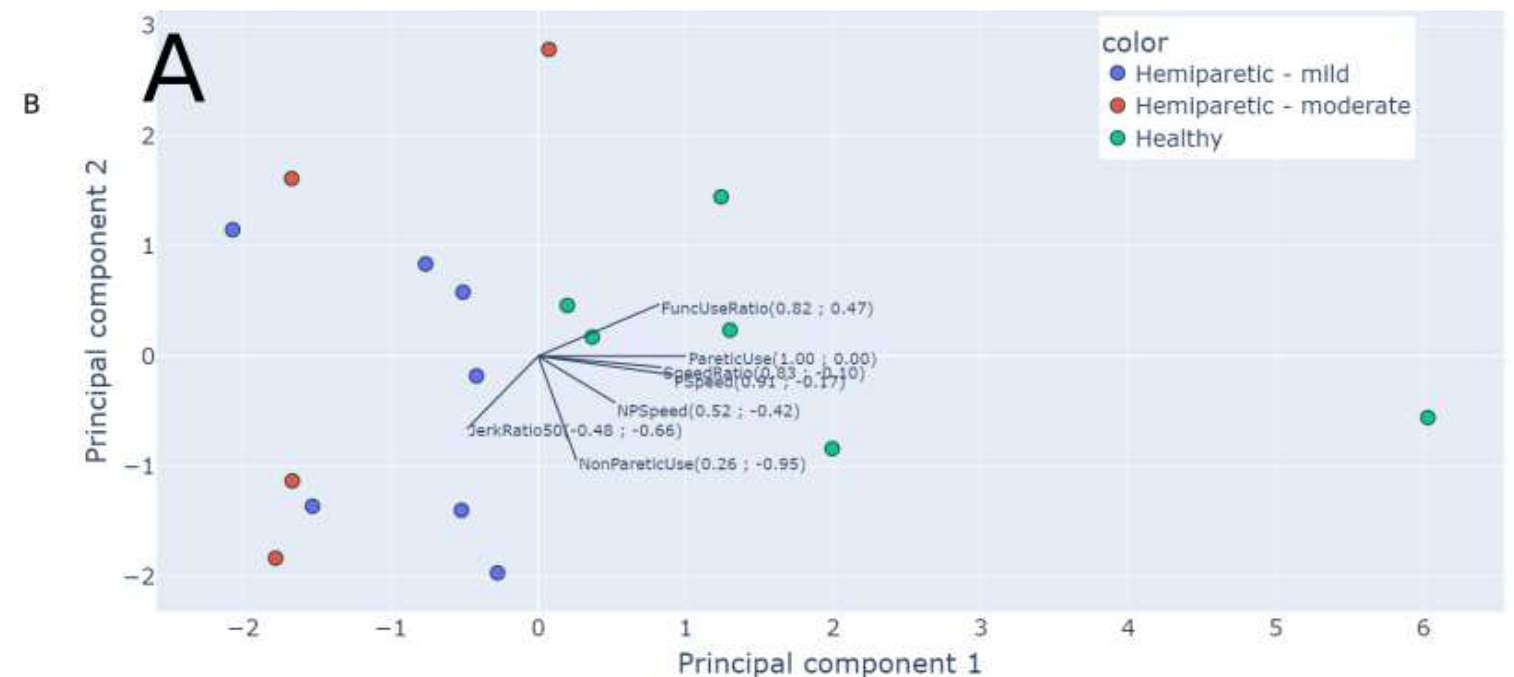

PC1 features importance (\%)

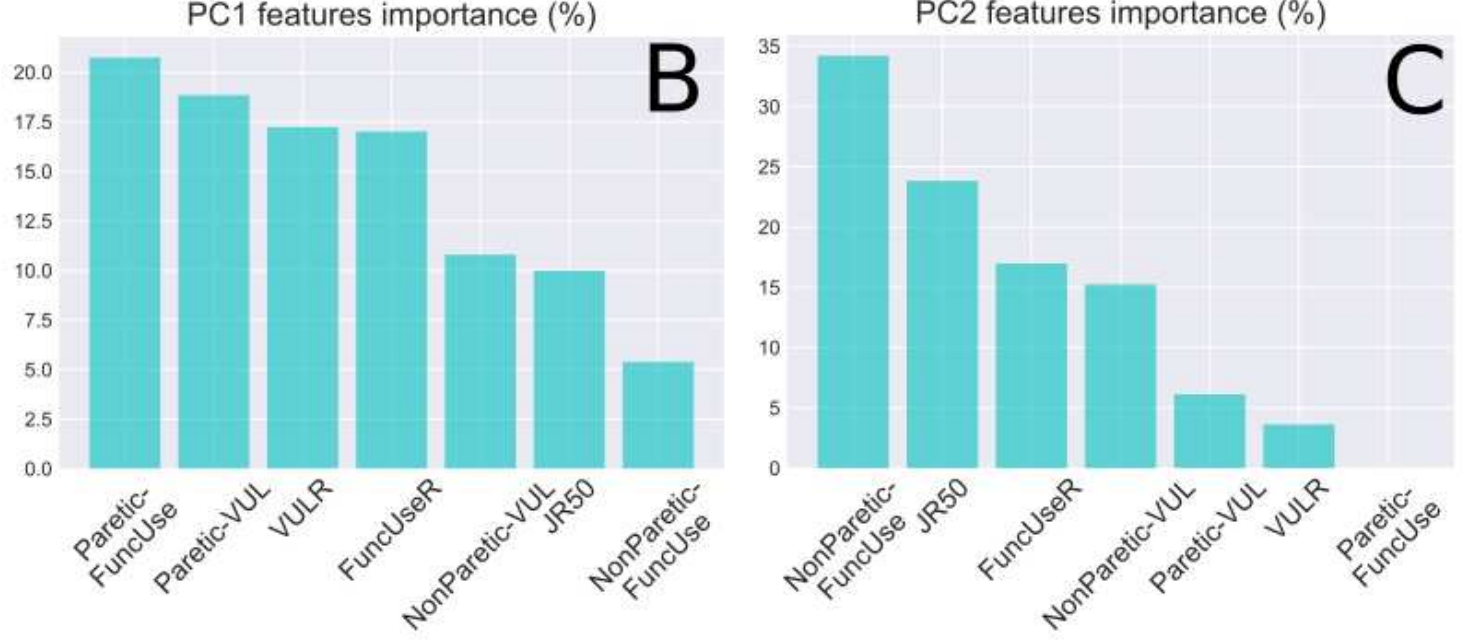

Figure 5 (A) Different biomarker principal component (PC) analysis scatter and loading plot in the PC1 and PC2 plane. Mild

294 (Fugl-Meyer>40), Moderate (Fugl-Meyer : [21-39]) stroke patients and healthy subjects are represented in blue, red and green 295 dots respectively. (B) PC1 features importance. (C) PC2 features importance.

\section{Discussion}

The aim of the study was to calculate multiple wrist actimetry biomarkers of stroke patients over a 7-days period in their home environment and then determine optimal biomarkers to

301 monitor functional paretic arm use (FuncUse). We performed, to our knowledge, the first study 15 
in stroke patients that calculated over an extended 7-days period multiple functional movement

303 biomarkers via two simple and lightweight wrists worn accelerometers, and compared these

304 values with values acquired in a healthy population. Accordingly, we derived new actimetry

305 biomarkers, in particular, we were able to calculate average elevation speed of execution of 306 functional movement (VUL) and the Jerk via the derivation of the elevation angle and the 307 acceleration respectively

308 Previous studies have measured the amount of functional movement of the upper limb 309 (FuncUse) in an ecological environment via IMUs placed at the wrist for a period of only 48 310 hours [Leuenberger et al, 2017]. According to our measurements, JR50 has a very low intra311 patient variability (standard deviation of plus or minus 0.05) but VULR and FuncUseR have 312 large standard deviations of up \pm 0.5 and \pm 0.3 respectively. It is then necessary to maximize 313 the number of measurement days to obtain relevant biomarker values. The arm elevation was 314 calculated using the same accelerometric metrics to which the authors added the calculation of 315 the yaw angle to identify movements in the horizontal plane. In our study, we choose to use 316 actimeters with a battery autonomy of more than one week for an acquisition frequency of 50 $317 \mathrm{~Hz}$ and thus to be more representative of the patient's ecological behavior. It is noted that 318 [Leuenberger et al, 2017] demonstrated a linear relationship between the Box and Blocks Test 319 and the ratio of movement of the paretic limb to the non-paretic limb.

320 However, they did not explore other biomarkers. These include average arm raise speed or 321 jerk ratio. In addition, they did not perform a comparison with a healthy population without 322 hemiparesis. A novel finding of the study was the significantly greater use of the non-dominant 323 limb of the healthy subjects compared to the paretic limbs of the stroke patients as well as a 324 significantly greater FuncUseR in the healthy subjects than in the stroke patients. Similarly, 
stroke subjects show significantly lower functional movement speeds and speed ratios than controls. Interestingly, a second novel finding was that the movement speed of the non-

327 paretic arm of the stroke patients was significantly slower than the dominant arm of the 328 healthy subject. The healthy subjects show on average three times more daily movement of 329 the non-dominant limb than the paretic limb of the stroke subjects. Indeed, healthy subjects 330 performed approximately 5000 functional movements per day with their non-dominant limb 331 whereas post-stroke patients realized only 1500 movements per day with their paretic limb. 332 Moreover, the healthy subjects show a FuncUseR close to 1, meaning an equal use of the 333 dominant and non-dominant upper limbs while the stroke patients show a very low median 334 FuncUseR close to 0.18 , which indicates 18 movements of the non-paretic limb for one 335 movement of the paretic limb. However, patients show an equivalent amount of functional 336 movement of the non-paretic limbs to that of the dominant limb of the volunteer subjects.

337 This suggests that the stroke patient studied here maintain a relatively normal amount of non338 paretic UL movement average.

339 The Jerk Ratio appears to reflect a ratio of the amount of movement in a given time frame 340 between the two limbs. While this ratio is balanced in healthy subjects, it shows a slight 341 imbalance in stroke subjects. These results show that there is a significantly higher probability 342 that stroke patients perform less movement, both functional and non-functional, with their 343 paretic limb than with their non-paretic limb when compared with the healthy population.

344 Furthermore, the study of correlations between the different biomarkers seems to show a 345 decreasing exponential relationship between the FuncUseR and the JR50. This suggests that 346 depending on the degree of deficit of the stroke patients, the two biomarkers would be 
complementary in establishing a diagnosis. Indeed, the FuncUseR seems to be more sensitive

348 for patients with upper limb behavior similar to healthy subjects, whereas the JR50 seems to

349 be more sensitive for subjects with significant hemiparesis (Figure 4.D). Furthermore, the

350 results showed that stroke patients had significantly lower average execution speeds of

351 functional movements than healthy subjects. It should be noted that the measured elevation

352 speeds seem to correspond to the values of the literature [Lacquaniti et al, 1982]. It is

353 interesting to note that there is a very strong positive correlation between the FuncUseRatio

354 and the VULR in healthy subjects but not in strokes patients. Finally, the principal component

355 analysis showed that the PC1 allows to differentiate with sufficient sensitivity the actimetric

356 results of healthy and hemiparetic subjects. We also see that the moderate hemiparetic

357 subjects have the lowest PC1 values.

358 In order to define a functional movement of the upper limbs we have arbitrarily chosen to define

359 an amplitude of elevation of the arm of more or less $30^{\circ}$. However, a large proportion of stroke

360 patients show uncontrolled flexion of the healthy elbow when walking. This phenomenon is

361 called "associated reaction" and may have an influence on the results of our study [Kahn et al,

362 2020]. This choice remains arbitrary and it would be necessary to explore the evolution of the

363 FuncUseR as well as the functional movement quantities as a function of this elevation

364 amplitude parameter. In particular, we would expect to observe no significant difference

365 between post-stroke and volunteers' subjects for functional movements of plus or minus $10^{\circ}$ of

366 elevation. Instead, the difference would tend to increase with the amplitude of the movement.

367 It would then be possible to identify an angular amplitude threshold value for each patient and

368 thus to obtain a new parameter allowing to better identify the patient deficiency. 
Another perspective would be to mix experimental method tools based on actimetry and artificial intelligence to identify with more precision what kind of movements is performed by 371 the patients [Sanhudo, 2021]. This identification of the movement will allow to better identify 372 the physical capacities of hemiparetic patients and thus to develop specific patient therapies. In 373 addition, other actimetric markers could be calculated to refine the study. In particular, we think 374 of the quantification of physical activity via the ENMO (Euclidean Norm Minus One) indicator 375 [White et al, 2016] as well as the quantification of smoothness during a functional movement via the study of [Melendez-Calderon et al, 2021]

The wrist actimetry methods developed in this article seems relevant for clinical use. Indeed, while the hemiparetic subjects studied had only mild or moderate deficit, some biomarkers were shown to be sensitive enough to identify significant differences between populations. It is now necessary to carry out an in-depth clinical study to identify different patient patterns, by enlarging the number of patients we involve and by covering a larger panel of different patients. While the FuncUseR developed by [Leumberger et al, 2017] correlates linearly with the BBT, 383 we do not know if this is the case for the FuncUseR developed in our study. Moreover, it would 384 be relevant to study the correlations of all the actimetric parameters present in our study with different clinical parameters. We are thinking in particular of the BBT and the Fugl Meyer score 386 for the upper limbs but also gait speed or 6 minutes walking test. Interestingly, the tool 387 developed in this article should make it possible to identify stroke patients with excellent 388 actimetric results. It would then be relevant to deepen the study by correlating actimetric and 389 clinical variables with other variables identifying motivation, environmental factors, anxiety and depression [Morris et al, 2013]. Such studies would allow the identification of other paths 391 for performance improvement. 
394 This study comparing healthy and post-stroke subjects found significant differences in 395 calculated actimetric biomarkers between healthy and post-stroke subjects. While the healthy 396 subjects had an upper extremity functional use ratio close to 1, the post-stroke subjects had a 397 ratio of about 0.2 . The post-stroke subjects do not seem to overuse their healthy limb to 398 compensate for the loss of motor skills in the paretic limb. The results of this study show the 399 interest of using different biomarkers for the longitudinal follow-up of patients with upper limb 400 hemiparesis.

401 - Ethics approval and consent to participate

403 The part of the study including post-stroke participants was approved by the IRB of the 404 Montpellier University Hospital, Montpellier, France (CPP SUD-EST II). The part of the 405 study including non-disabled healthy participants was approved by the IRB of the University 406 of Montpellier, France. All participants gave their informed consent for participating the 407 study.

\section{- Consent for publication}

Not applicable.

\section{- Availability of data and materials}

411 The datasets used and/or analysed during the current study are available from the 412 corresponding author on reasonable request. 


\section{- Funding :} The study was in part supported by the Direction générale de l'offre de soins (DGOS) in the French Ministère des Affaires Sociales et de la Santé (Programme hospitalier de recherche infirmière et paramédicale- PHRIP-2018-0731).

\section{- Authors' contributions}

GD: conceptualization of model and computational framework, software, formal analysis, data collection and curation, writing — original draft. DM: Conceptualization and design of the study, results interpretation, writing - review and editing. MM: Results interpretation writing - review and editing . IL: Writing - review and editing. KB: Conceptualization and design of the study, data collection, results interpretation, writing - review and editing. All authors read and approved the final manuscript.

\section{- Acknowledgements}

428 The authors would like to thank Thomas Bonnet for his help in clinical experimentation.

429 Special thank goes also to all participants for taking part in the study. 


\section{References}

437 Murray, C. J., Vos, T., Lozano, R., Naghavi, M., Flaxman, A. D., Michaud, C., ... \& Haring, D. 438 (2012). Disability-adjusted life years (DALYs) for 291 diseases and injuries in 21 regions, 439 1990-2010: a systematic analysis for the Global Burden of Disease Study 2010. The lancet, $440 \quad 380(9859), 2197-2223$

442 Morris, J. H., Van Wijck, F., Joice, S., \& Donaghy, M. (2013). Predicting health related quality 443 of life 6 months after stroke: the role of anxiety and upper limb dysfunction. Disability and 444 rehabilitation, 35(4), 291-299.

446 Kwakkel, G., Kollen, B. J., van der Grond, J., \& Prevo, A. J. (2003). Probability of regaining 447 dexterity in the flaccid upper limb: impact of severity of paresis and time since onset in acute 448 stroke. Stroke, 34(9), 2181-2186. 
450 Fugl-Meyer, A. R., Jääskö, L., Leyman, I., Olsson, S., \& Steglind, S. (1975). The post-stroke

451 hemiplegic patient. 1. a method for evaluation of physical performance. Scandinavian journal 452 of rehabilitation medicine, 7(1), 13-31.

453

454 McLaren, R., Signal, N., Lord, S., Taylor, S., Henderson, J., \& Taylor, D. (2020). The volume 455 and timing of upper limb movement in acute stroke rehabilitation: still room for 456 improvement. Disability and rehabilitation, 42(22), 3237-3242.

457

458 Bailey, R. R., \& Lang, C. E. (2014). Upper extremity activity in adults: referent values using 459 accelerometry. Journal of rehabilitation research and development, 50(9), 1213.

460

461 Bailey, R. R., Klaesner, J. W., \& Lang, C. E. (2015). Quantifying real-world upper-limb activity 462 in nondisabled adults and adults with chronic stroke. Neurorehabilitation and neural repair, $46329(10), 969-978$.

464

465 White, T., Westgate, K., Wareham, N. J., \& Brage, S. (2016). Estimation of physical activity 466 energy expenditure during free-living from wrist accelerometry in UK adults. PLoS One, $467 \quad 11(12), \mathrm{e} 0167472$. 
469

470

471

472

473

474

475

476

477

478

479

480

481

483

484

485

486

487

488

489

Leuenberger, K., Gonzenbach, R., Wachter, S., Luft, A., and Gassert, R. (2017). A method to qualitatively assess arm use in stroke survivors in the home environment. Medical \& biological engineering \& computing, 55(1):141-150.

Pan, Y. C. P., Goodwin, B., Sabelhaus, E., Peters, K. M., Bjornson, K. F., Pham, K. L., ... \& Steele, K. M. (2020). Feasibility of using acceleration-derived jerk to quantify bimanual arm use. Journal of neuroengineering and rehabilitation, 17(1), 1-8.

Melendez-Calderon, A., Shirota, C., \& Balasubramanian, S. (2021). Estimating movement smoothness from inertial measurement units. Frontiers in bioengineering and biotechnology, 8,1507

Sanhudo, L., Calvetti, D., Martins, J. P., Ramos, N. M., Mêda, P., Gonçalves, M. C., \& Sousa, H. (2021). Activity classification using accelerometers and machine learning for complex construction worker activities. Journal of Building Engineering, 35, 102001.

Lang, C. E., Strube, M. J., Bland, M. D., Waddell, K. J., Cherry-Allen, K. M., Nudo, R. J., ... \& Birkenmeier, R. L. (2016). Dose response of task-specific upper limb training in people at least 6 months poststroke: A phase II, single-blind, randomized, controlled trial. Annals of neurology, 80(3), 342-354. 
491 Waddell, K. J., Strube, M. J., Bailey, R. R., Klaesner, J. W., Birkenmeier, R. L., Dromerick, A. 492 W., \& Lang, C. E. (2017). Does task-specific training improve upper limb performance in daily 493 life poststroke?. Neurorehabilitation and neural repair, 31(3), 290-300.

494

495 Lacquaniti, F., \& Soechting, J. F. (1982). Coordination of arm and wrist motion during a 496 reaching task. Journal of Neuroscience, 2(4), 399-408.

497

498 Bleecker, M. L., Bolla-Wilson, K., Kawas, C., \& Agnew, J. (1988). Age-specific norms for 499 the mini-mental state exam. Neurology, 38(10), 1565-1565.

500

501 Kahn, M. B., Williams, G., Mentiplay, B. F., Bower, K. J., Olver, J., \& Clark, R. A. (2020). 502 Quantification of abnormal upper limb movement during walking in people with acquired brain 503 injury. Gait \& Posture, 81, 273-280.

504

505

506 